\title{
Ethnicity-stratified analysis of the association between XRCC3 Thr241Met polymorphism and leukemia: an updated meta-analysis
}

\author{
Zhengjun Xie*, Wei Peng, Qiuhua Li, Wei Cheng and Xin Zhao
}

\begin{abstract}
Background: Presently, whether X-ray repair cross complementing group 3 (XRCC3) Thr241Met polymorphism is correlated to leukemia risk remains controversial. Because of this reason, the objective of current study is to explore whether XRCC3 Thr241Met polymorphism confers risk to leukemia.

Methods: Two independent authors systematically and comprehensively searched Pubmed, Embase, the Cochrane library, Google academic, China National Knowledge Infrastructure (CNKI). Search time is from database foundation to March 2021.

Results: Overall, significant associations between leukemia risk and XRCC3 Thr241 Met polymorphism were found in Caucasian population by allele contrast (T vs. C: OR 1.20, 95\% Cl 1.02-1.40), homozygote comparison (TT vs. CC: OR $1.35,95 \% \mathrm{Cl} 1.05-1.73$ ), and recessive genetic model (TT vs. TC/CC: OR 1.31, 95\% Cl 1.04-1.64).

Conclusions: The present meta-analysis suggests that the XRCC3 Thr241Met polymorphism may be a risk factor for leukemia in Caucasian population.
\end{abstract}

Keywords: Leukemia, Genetic polymorphism, XRCC3, Meta-analysis

\section{Background}

Leukemia is a very frequent malignance tumor originating from hematopoietic stem cells. The leukemia cell stops at different stages of cell development due to uncontrolled proliferation, dysdifferentiation and aleukemiatosis block. Its common symptoms are anemia, infection and bleeding. The incidence of leukemia in China is approximately $3-8$ individuals per $100,000[1,2]$. About One hundred thousand people are diagnosed with leukemia every year [2]. There is no doubt that the occurrence of leukemia brings a huge burden on individuals, families, and health care systems. However, its exact etiology and pathogenesis remains unknown.

*Correspondence: zhj_xie@hotmail.com

Department of Hematology, The Fifth Affiliated Hospital of Zunyi Medical University, Zhufeng Avenue 1439, Zhuhai 519000, China
Several studies have shown that the occurrence of leukemia is associated with exposure to risk environment factors such as benzene, formaldehyde, smoking history, residence decoration and the use of different kinds of hair dye. Benzene and its metabolites make bone marrow damaged by immune-mediated responses, leading to the occurrence of leukemia [3]. A large-scale cohort study has shown that exposure to environmental benzene is associated with a variety of hematological malignancies, including acute leukemia, MDS, and T-cell lymphoma [4]. With the increased dose and frequency of hair colorants, the micronucleus rate of polychromatic erythrocytes (PCE) in bone marrow of mice increased, suggesting hair colorants can cause chromosomal damage, and long-term use of hair colorants will increase the risk of acute leukemia. Indoor decoration materials can release hundreds of pollutants such as benzene, 
formaldehyde, radon and volatile harmful gases. A number of epidemiological studies have shown that short occupancy time after decoration is associated with the incidence of leukemia [5].

The occurrence and development of leukemia is a complicated process. Many scholars attribute it to some risk factors including physical factors, chemical factors, and virus infection; however, these factors are not acting as a necessary role for leukemia occurrence and progression. Approximate 30\% patients do not embrace physical factors, chemical factors, and virus infection will acquire leukemia. All the above evidences indicate that extra genetic or non-genetic factors modulating leukemia susceptibility are yet to be identified.

Although leukemia pathogenesis is an extremely complicated process and the exact pathogenesis of leukemia is still unknown, studies have shown that DNA damage is closely related to its occurrence and development [6]. Many researchers have shown that multiple forms of DNA damage can occur and double-stranded DNA breakage is the most common type, leading to cell death, loss of genetic material, and translocation or deletion of chromosomes. On the other hand, there are many complex mechanisms in the body to maintain the stability of genetic material, including DNA repair pathways, antioxidant stress systems and anti-damage factor systems [7]. Double-stranded fracture repair is a form of DNA repair pathway, which also includes homologous recombination repair and non-homologous recombination repair [8]. XRCC3 is an important protein during the process of DNA homologous recombination repair, and its single nucleotide polymorphisms play an important role on DNA homologous recombination repair $[9,10]$. Some studies have suggested that XRCC3 Thr241Met polymorphism is associated with leukemia risk. But other studies hold the controversial idea.

Yan et al. published a literature in 2014 that also investigates the association between XRCC3 Thr241Met polymorphism and leukemia risk [11]. Regrettably, only seven studies were included in their meta-analysis. They concluded that XRCC3 Thr241Met polymorphism was not associated with leukemia risk. Qin et al. published a literature in 2013 that also investigates the association between XRCC3 Thr241Met polymorphism and leukemia risk [12]. Similarly, they also get a negative result. Compared with the previous meta-analysis, some important advantages of our paper should be pointed out. Firstly, more eligible studies were enrolled in our metaanalysis. By this means, 16 literatures (10 Caucasian, 4 Asian, and 2 African) were included. Compared with previous meta-analysis, the number of eligible literatures greatly increased. And the merit of meta-analysis is just improving statistical efficiency and making the results more truthful. What's more, the present study reverses the previous results. We have first discovered that XRCC3 Thr241Met polymorphism contributes an increased risk to leukemia of Caucasian population. The results of our study indicate the limited sample size of previous meta-analysis. So that we think the present meta-analysis is reliable and comprehensive.

As far as we know, this is the first meta-analysis which comprehensively explores the association between XRCC3 Thr241Met polymorphism and leukemia susceptibility. The objective of current study is to estimate whether XRCC3 Thr241Met polymorphism confers risk to leukemia.

\section{Materials and methods \\ Search strategy}

Two independent authors systematically and comprehensively searched Pubmed (https://www.ncbi.nlm.nih.gov/ pubmed/), EMBASE (https://www.embase.com/), the Cochrane library(https://www.cochranelibrary.com/), Google academic (https://scholar.google.com/), and Chinese national knowledge internet (https:// www.cnki. net/). Search time is from database foundation to March 2021. The keywords applied in the search process were as follows: ("XRCC3" or "X-ray repair cross complementing group 3") together with ("leukemia"). The literature language was limited to English language and Chinese language. Additionally, in order to avoid the omission of relevant literatures, we searched the references as much as possible.

\section{Inclusion and exclusion criteria}

The inclusion criteria must meet a series of conditions: (a) a case-control study; (b) making an assessment of the association between XRCC3 Thr241Met polymorphism and leukemia risk; (c) offering sufficient information and data to count OR and 95\%CI. The exclusion criteria also must meet a few conditions: (a) patients with other hematological system diseases such as multiple myeloma, aplastic anemia, myelodysplastic syndrome, autoimmune hemolytic anemia, idiopathic thrombocytopenic purpura. (b) patients with some inflammatory diseases or cardiovascular and cerebrovascular diseases such as urinary tract infection or shock, acute myocardial infarction or unstable angina, rheumatoid arthritis or systemic lupus erythematosus. (c) not offering sufficient data for meta-analysis. (d) its experiment objective was pig, rat or other animals.

\section{Data extraction and methodological quality assessment}

All the necessary information was independently reviewed and assessed by first author and second author (Zhengjun Xie; Wei Peng). Then this contradictory 
data or information was reassessed by the third author (Qiuhua Li). The extracted data consisted of author name, publication year, genotyping methods, sample size, ethnicity, matching criteria, source of control, HWE conformity. If the similar opinion could not reach in the course of data extraction, suggestion was offered by another experienced researcher (Qiuhua Li) to determine the correct selection. The similar method was applied equally to evaluation of literature quality. In the present meta-analysis, we applied the risk assessment criteria of Newcastle-Ottawa Scale (NOS) bias to evaluate the quality of each literature. The main criteria consisted of three aspects including selection of enrolled study subjects (0-4 scores); between-group comparability (0-2 scores); exposure outcomes and factors ( $0-3$ scores). It should be noted that the ethics approval of our study was waived by Ethics Committee of The Fifth Affiliated Hospital of Zunyi Medical University as no human or animal was directly enrolled in our study and meta-analysis is the statistical analysis of large collection of analysis results from individual studies for the purpose of integrating the findings.

\section{Statistical analysis}

The association power was assessed through the corresponding indexes including OR and 95\%CI. And both the Q-statistic and $\mathrm{I}^{2}$ statistics would be applied [13]. Four genetic models were applied in the present metaanalysis including allele contrast ( $\mathrm{T}$ vs. $\mathrm{C}$ ), homozygote comparison (TT vs. CC), heterozygous comparison (TC vs. $\mathrm{CC}$ ), recessive genetic model (TT vs. TC/CC) and dominate genetic model (TT/TC vs. CC). The model of fixed-effects and random-effects would be put into use on the basis of heterogeneity degree $[14,15]$. $\mathrm{I}^{2}<50 \%$ was considered to low heterogeneity, $50 \leq \mathrm{I}^{2}<75 \%$ was considered to moderate heterogeneity and $\mathrm{I}^{2} \geq 75 \%$ was considered to significant heterogeneity. If $\mathrm{I}^{2}<50 \%$ and $\mathrm{P}>0.1$, the fixed-effects model would be used. If $\mathrm{I}^{2} \geq 50 \%$ or $\mathrm{P} \leq 0.1$, the random -effects model would be used. Furthermore, the Galbraith plot was used to spot the outliers to find out the potential heterogeneity as much as possible. Sensitive analysis was applied to detect the influential studies which might contribute obvious bias to final results. The funnel plot and Egger's test were put into use to recognize the existence of publication bias [16]. Metaregression and subgroup analysis were used to detect and deal with the possible source of heterogeneity. The Stata 12.0 would be responsible for the whole statistics. The meta-analysis was conducted based on the Preferred Reporting Items for Systematic Reviews and Meta-Analyses (PRISMA) 2009 checklist (Additional file 1: Table S1 Checklist) [17, 18]. Furthermore, HWE conformity was based on the $\mathrm{P}$ value of control group ( $\mathrm{P}>0.05$ was considered HWE conformity).

\section{Results \\ General information}

PRISMA 2009 Flow Diagram shows the flow chart of meta-analysis search course (Additional file 2: Table S2 Checklist) $[17,18]$. Based on the search strategy, sixteen literatures were satisfactory [19-34]. Table 1 shows the detailed information of all literatures. In total, sixteen literatures consisted of ten literatures from European countries and America, four literatures from Asian countries and two literatures from African countries. Different genotyping methods were used such as direct sequencing, PCR-RFLP and TaqMan. The publication year ranged from 2002 to 2018 and the controls were populationbased or hospital-based. All the genotyping frequency of controls was conform to HWE. And the sample size ranged from 80 to 1600 .

\section{Meta-analysis results}

The meta-analysis results between XRCC3 Thr241Met polymorphism and leukemia susceptibility are shown in Table 2. Generally, positive finding between leukemia and XRCC3 Thr241Met polymorphism was found in Caucasian population by allele contrast ( $\mathrm{T}$ vs. C: OR $1.20,95 \%$ CI 1.02-1.40, $\mathrm{P}=0.026$, Fig. 1), homozygote comparison (TT vs. CC: OR 1.35, 95\% CI 1.05-1.73, P=0.018, Fig. 2), and recessive genetic model (TT vs. TC/CC: OR 1.31, 95\% CI 1.04-1.64, P=0.023, Fig. 3).

\section{Evaluation of heterogeneity and sensitivity}

Significant heterogeneity was found under all the allele contrast $\left(X^{2}=141.02, \mathrm{P}=0, \mathrm{I}^{2}=86.5\right.$, Table 2$)$, homozygote comparison $\left(\chi^{2}=59.73, P=0, \mathrm{I}^{2}=68.2\right.$, Table 2$)$, recessive genetic model $\left(\chi^{2}=55.58, \mathrm{P}=0, \mathrm{I}^{2}=65.8\right.$, Table 2), and dominate genetic model $\left(\chi^{2}=109.36\right.$, $\mathrm{P}=0, \mathrm{I}^{2}=82.6$, Table 2 ). To detect the possible source of heterogeneity, we conducted meta-regression and subgroup analysis. Meta-regression revealed that ethnicity was the main source of heterogeneity which contributed substantial heterogeneity to the final results. Then we conduct subgroup analyses stratified by ethnicity. Subsequently, the heterogeneity reduced in Caucasian population under allele contrast $\left(\chi^{2}=27.38\right.$, $\mathrm{P}=0.004, \mathrm{I}^{2}=59.8$, Table 2$)$, homozygote comparison $\left(X^{2}=15.69, P=0.153, I^{2}=29.9\right.$, Table 2$)$, recessive genetic model $\left(X^{2}=15.30, P=0.169, \mathrm{I}^{2}=28.1\right.$, Table 2$)$, and dominate genetic model $\left(\chi^{2}=23.28, P=0.016\right.$, $\mathrm{I}^{2}=52.8$, Table 2). In order to further detect the source of heterogeneity of African and Asian population, we conduct Galbraith plots to find out the outliers which might influence the heterogeneity. Consequently, we 


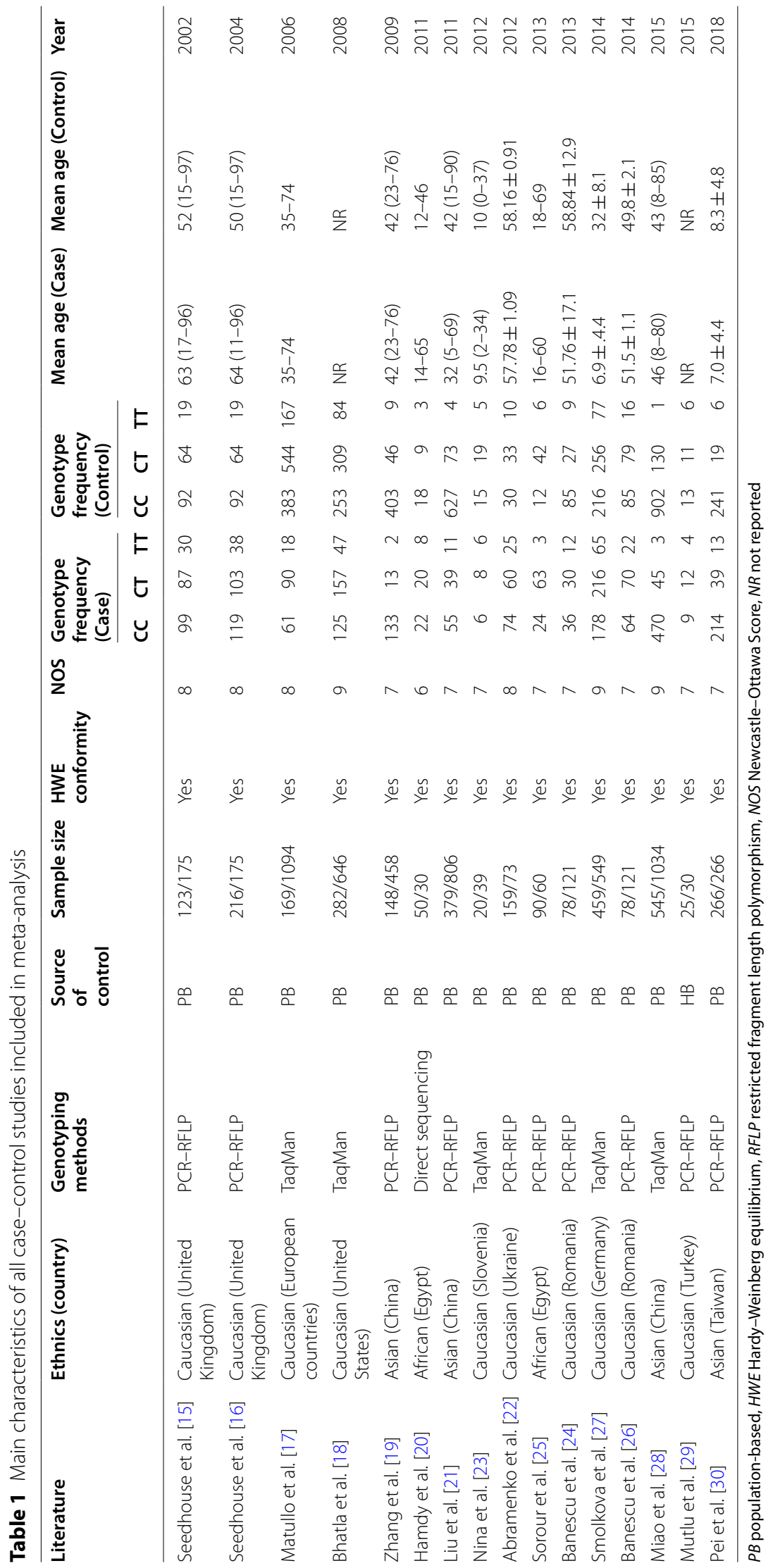


Table 2 The general results for the association between XRCC3 Thr241Met polymorphism with leukemia risk

\begin{tabular}{|c|c|c|c|c|c|c|c|c|c|}
\hline \multirow[t]{2}{*}{ Comparison } & \multirow[t]{2}{*}{ Group } & \multirow[t]{2}{*}{$\mathrm{N}$} & \multicolumn{3}{|c|}{ Test of association } & \multirow[t]{2}{*}{ Mode } & \multicolumn{3}{|c|}{ Test of heterogeneity } \\
\hline & & & OR & $95 \% \mathrm{Cl}$ & $P$ & & $\overline{x^{2}}$ & $P$ & $1^{2}$ \\
\hline \multirow[t]{4}{*}{ T versus. $C$} & Overall & 20 & 1.21 & $1.00-1.47$ & 0.049 & Random & 96.21 & 0 & 80.3 \\
\hline & Caucasian & 12 & 1.20 & $1.02-1.40$ & 0.026 & Random & 27.38 & 0.004 & 59.8 \\
\hline & Asian & 5 & 1.25 & $0.62-2.50$ & 0.530 & Random & 49.34 & 0 & 91.9 \\
\hline & African & 3 & 0.91 & $0.52-1.58$ & 0.727 & Random & 5.50 & 0.064 & 63.6 \\
\hline \multirow[t]{4}{*}{ TT versus. CC } & Overall & 20 & 1.39 & $1.04-1.86$ & 0.027 & Random & 37.78 & 0.006 & 49.7 \\
\hline & Caucasian & 12 & 1.35 & $1.05-1.73$ & 0.018 & Fixed & 15.69 & 0.153 & 29.9 \\
\hline & Asian & 5 & 2.05 & $0.74-2.66$ & 0.169 & Random & 8.44 & 0.077 & 52.6 \\
\hline & African & 3 & 0.51 & $0.12-2.16$ & 0.361 & Random & 5.65 & 0 & 64.6 \\
\hline \multirow[t]{4}{*}{ TC versus. CC } & Overall & 20 & 1.05 & $0.84-1.34$ & 0.443 & Random & 32.99 & 0.005 & 55.8 \\
\hline & Caucasian & 12 & 1.07 & $0.86-1.32$ & 0.123 & Fixed & 11.22 & 0.166 & 30.4 \\
\hline & Asian & 5 & 1.01 & $0.54-1.76$ & 0.643 & Random & 9.62 & 0.078 & 54.8 \\
\hline & African & 3 & 1.25 & $0.77-1.98$ & 0.255 & Random & 7.32 & 0.002 & 62.1 \\
\hline \multirow[t]{4}{*}{ TT versus. $\mathrm{TC}+\mathrm{CC}$} & Overall & 20 & 1.31 & $0.99-1.73$ & 0.063 & Random & 39.41 & 0.004 & 51.8 \\
\hline & Caucasian & 12 & 1.31 & $1.04-1.64$ & 0.023 & Fixed & 15.30 & 0.169 & 28.1 \\
\hline & Asian & 5 & 1.95 & $0.71-5.37$ & 0.194 & Random & 8.42 & 0.078 & 52.5 \\
\hline & African & 3 & 0.45 & $0.12-1.69$ & 0.239 & Random & 5.52 & 0.063 & 63.8 \\
\hline \multirow[t]{4}{*}{$\mathrm{TT}+\mathrm{TC}$ versus. $\mathrm{CC}$} & Overall & 20 & 1.19 & $0.99-1.43$ & 0.071 & Random & 49.23 & 0 & 61.4 \\
\hline & Caucasian & 12 & 1.18 & $0.97-1.44$ & 0.104 & Random & 23.28 & 0.016 & 52.8 \\
\hline & Asian & 5 & 1.19 & $0.69-2.05$ & 0.537 & Random & 22.31 & 0 & 82.1 \\
\hline & African & 3 & 1.03 & $0.57-1.87$ & 0.928 & Random & 2.81 & 0.246 & 28.8 \\
\hline
\end{tabular}

Bold values emphasize $\mathrm{P}<0.05$

OR odds ratio, $95 \% \mathrm{Cl} 95 \%$ confidence interval

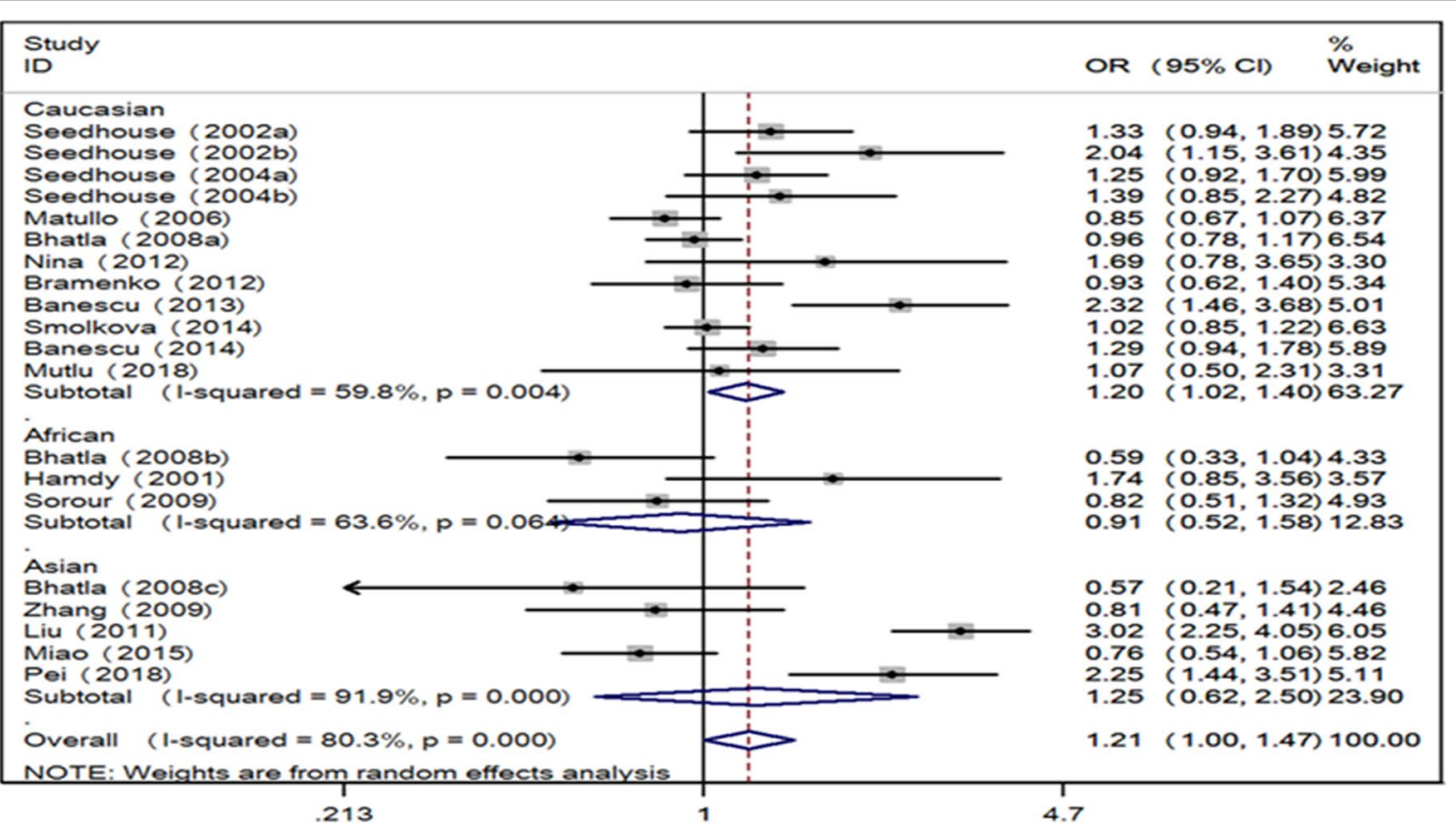

Fig. 1 Forest plot for the associations between XRCC3 Thr241Met polymorphism and leukemia risk through allele contrast (T vs. C). XRCC3 X-ray repair cross complementing group 3, OR odds ratio, Cl confidence interval 


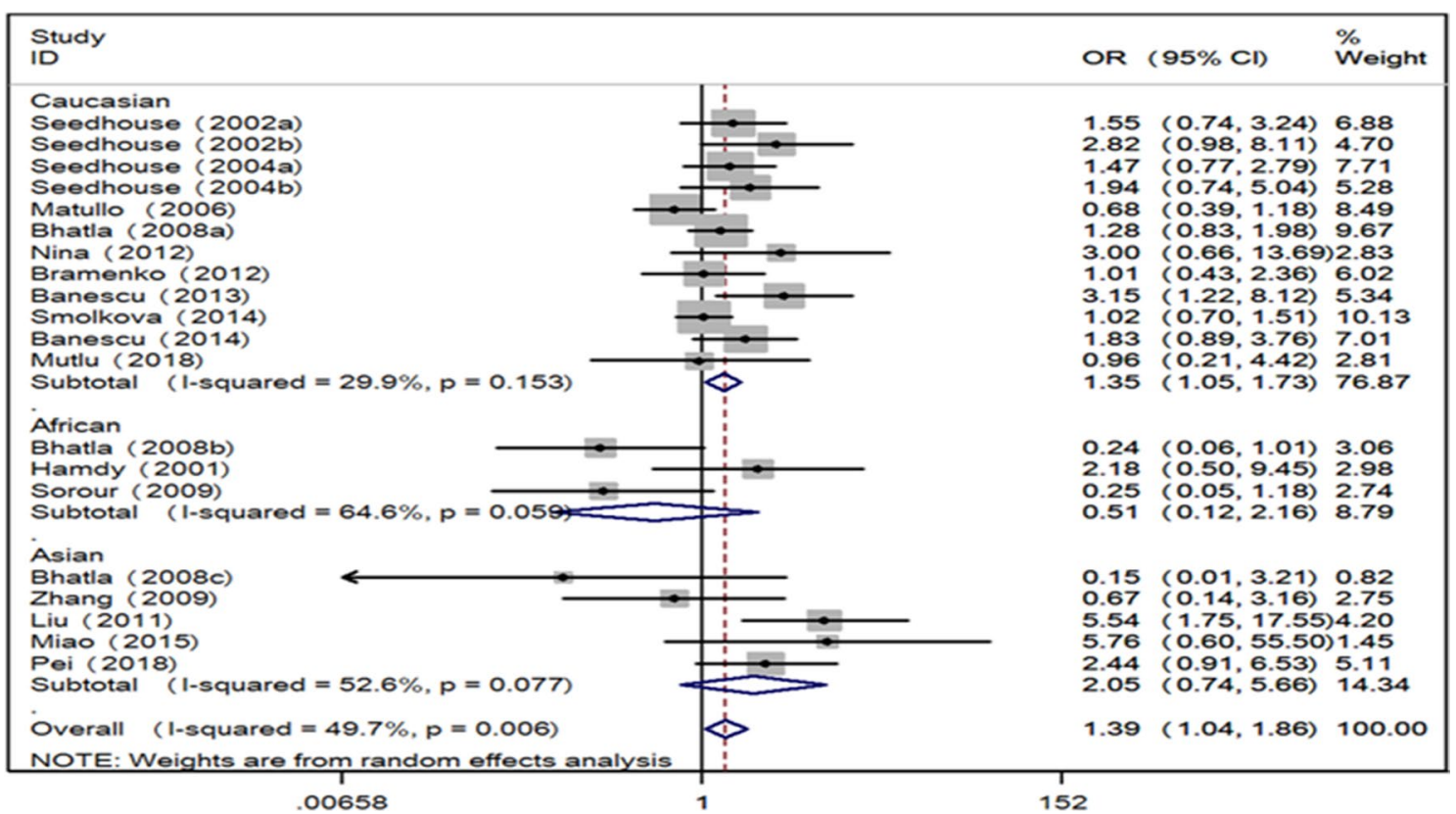

Fig. 2 Forest plot for the associations between XRCC3 Thr241Met polymorphism and leukemia risk through homozygote comparison (TT vs. CC). XRCC3, X-ray repair cross complementing group 3; OR odds ratio, Cl confidence interval

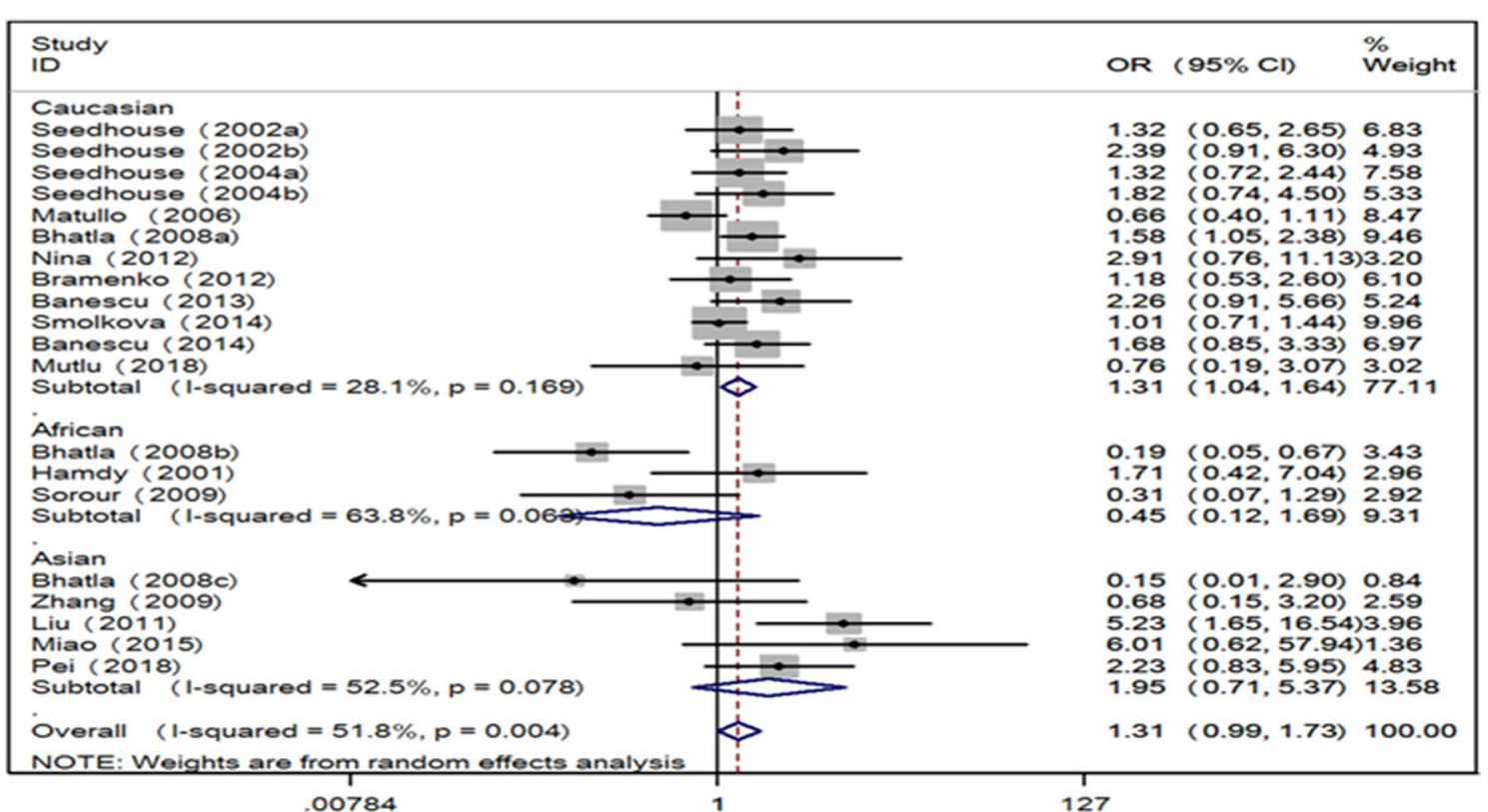

Fig. 3 Forest plot for the associations between XRCC3 Thr241Met polymorphism and leukemia risk through recessive genetic model (TT vs. TC/CC). XRCC3 $\mathrm{X}$-ray repair cross complementing group 3, OR odds ratio, Cl confidence interval

found the studies Liu et al. and Hamdy et al. were not within reasonable limits (Fig. 4). Then we excluded two studies and performed meta-analysis again, we found that the results were not altered.

\section{Sensitivity analysis and Publication Bias}

To verify the reliability and stability of meta-analysis results, sensitive analysis was applied to detect the influential studies which might contribute obvious bias to 


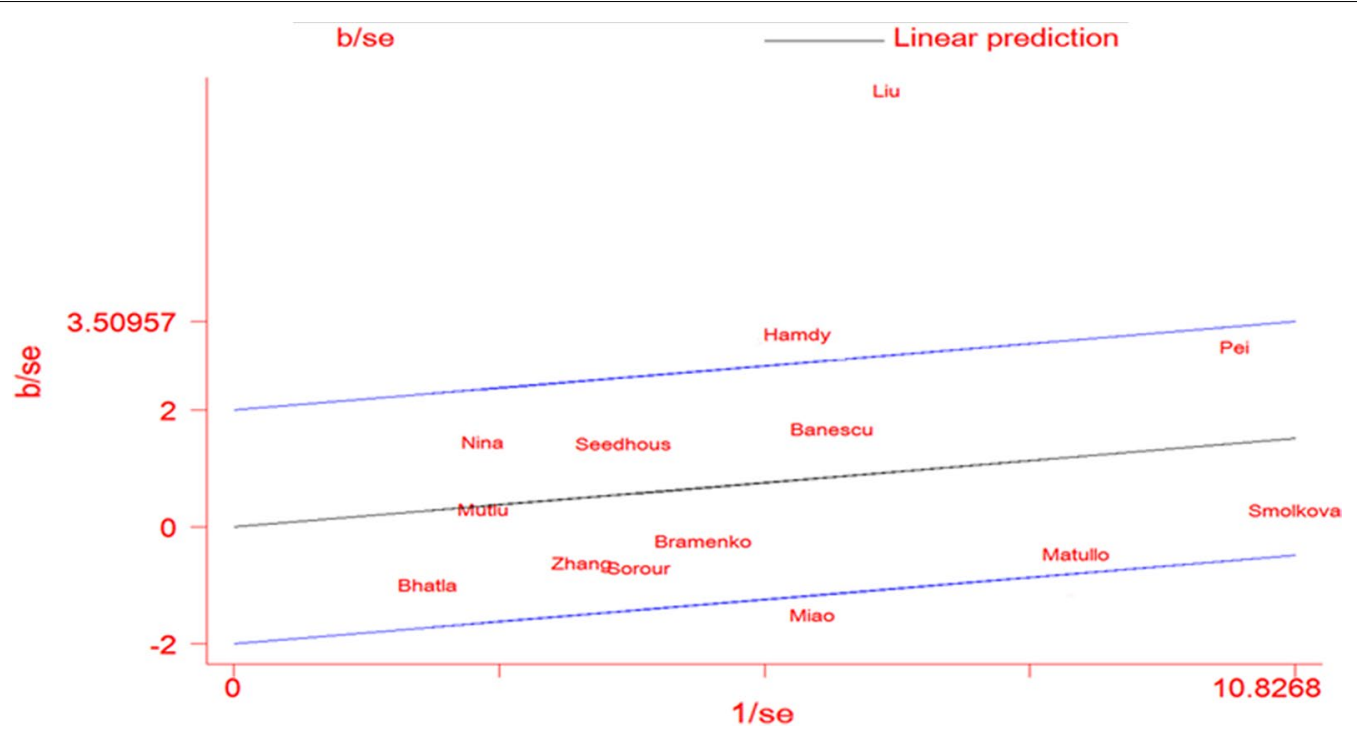

Fig. 4 Galbraith plot of XRCC3 Thr241Met polymorphism and leukemia risk by allele contrast: A vs. G. XRCC3 X-ray repair cross complementing group 3

final results. The final results were not altered by any single literature, suggesting that the results of our metaanalysis were stable and reliable (Fig. 5).We only find mild asymmetrical by funnel plot $(\mathrm{P}=0.881)$ (Fig. 6 and Additional file 3: Figure S3). And we do not find any evident publication bias by Egger's test in any genetic model $(\mathrm{P}=0.486,0.682,0.514,0.407,0.357$, respectively $)$.

\section{Discussion}

Considering the increasing prevalence of leukemia and its percentage among population death causes, leukemia prevention and treatment are always one of the key medical research subjects in all countries. The morbidity is the highest in some developing countries including China, Iran, Thailand, Pakistan, Mexico and Latin America, and

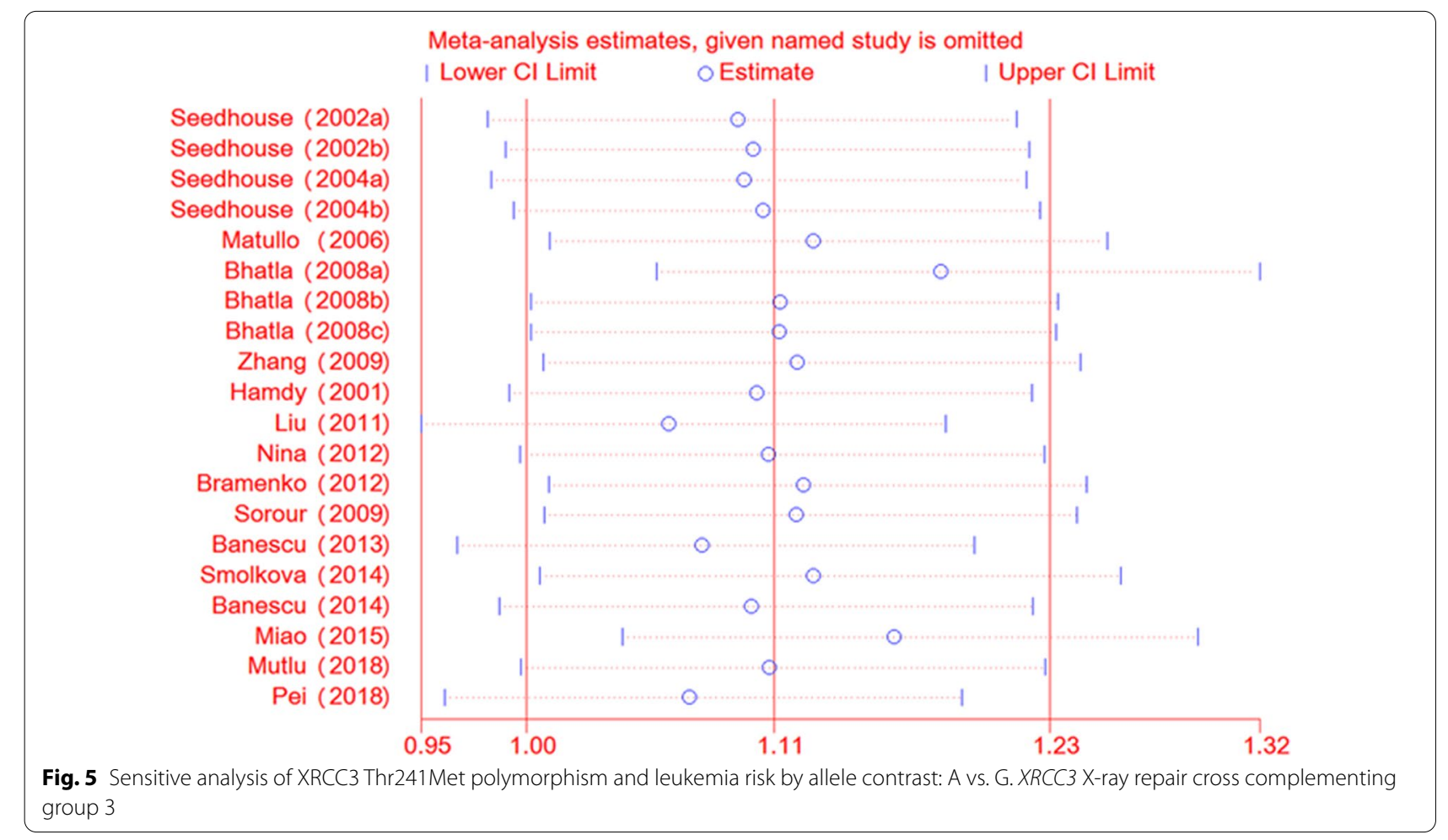




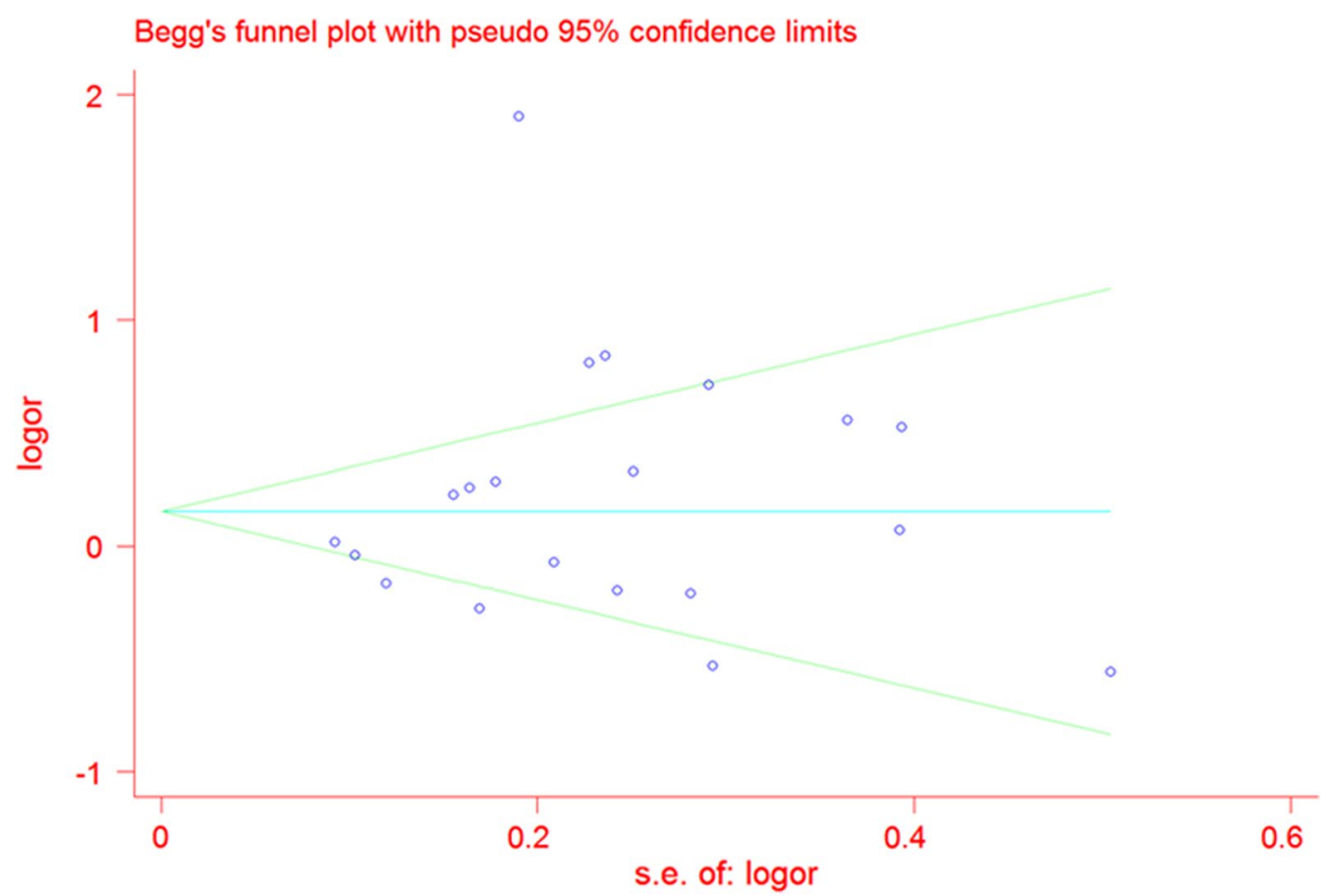

Fig. 6 Funnel plot on publication bias for the associations between XRCC3 Thr241Met polymorphism and susceptibility to leukemia through the allele model (T vs. C). XRCC3 X-ray repair cross complementing group 3, SE standard error, OR odds ratio

the morbidity can reach $2.5-8$ individuals per 100,000 [35-42]. The leukemia not only poses a threat to People's health and lives but also brings huge economic burden and mental pressure to the society and families. Nevertheless, it is well-established that the etiology of leukemia is awfully complicated and the role of etiology remains to be elucidated.

Although the pathogenesis of leukemia is a complex process, one thing for sure is that its pathogenesis is mainly caused by the comprehensive effects of environmental factors and genetic factors. The environmental factors consist of some harmful substances including ionizing radiation, benzene, mercury, and other pernicious elements. Moreover, long-term hair color, virus infection and long-term use of antibiotics are also harmful and contribute to the occurrence of leukemia.

Apart from these non-genetic risk factors, genetic factors play a vital role in pathogenesis of leukemia. Compared with African-American women, the leukemia morbidity of Latinos and whites is $4-5$ times greater. Moreover, the white race suffers from a higher morbidity than the black race and Spanish characters. These results indicate that genetic factors are crucial for leukemia pathogenesis. As far as we know, this is the first meta-analysis which comprehensively explores the association between XRCC3 Thr241Met polymorphism and leukemia susceptibility. It should be noted that Yan et al. published a literature in 2014 that also investigates the association between XRCC3 Thr241Met polymorphism and leukemia risk [11]. Regrettably, only seven studies were included in their meta-analysis. They concluded that XRCC3 Thr241Met polymorphism was not associated with leukemia risk. Qin et al. published a literature in 2013 that also investigates the association between XRCC3 Thr241Met polymorphism and leukemia risk [12]. Similarly, they also get a negative result. Compared with the previous meta-analysis, some important advantages of our paper should be pointed out. Firstly, more eligible studies were enrolled in our meta-analysis. By this means, 16 literatures (10 Caucasian, 4 Asian, and 2 African) were included. Compared with previous meta-analysis, the number of eligible literatures greatly increased. And the merit of meta-analysis is just improving statistical efficiency and making the results more truthful. What's more, the present study reverses the previous results. We have first discovered that XRCC3 Thr241Met polymorphism contributes an increased risk to leukemia of Caucasian population. The results of our study indicate the limited sample size of previous metaanalysis. So that we think the present meta-analysis is reliable and comprehensive.

We found that XRCC3 Thr241Met polymorphism contributes no risk to leukemia of African and Asian population but contributes an increased risk to leukemia of 
Caucasian population. To be specific, the $\mathrm{T}$ allele and TT genotype were risk factors and they contribute an increased risk to leukemia in Caucasian population. For the past few years, N4-acetylcytidine (ac4C) has been subject to widespread attention as comprehensive modifications have been detected in mRNAs of human and yeast [43]. It contributes to accurately reading codons in the process of translation and improving translational efficiency [43]. Furthermore, there is a direct correlation between ac4C and occurrence, development, progression of number diseases [43].

Eliminating the source of bias is of vital importance for gene polymorphism association meta-analysis. Hence, we have attempted to conduct all the three patterns in the present meta-analysis. Firstly, allele contrast was used to find out the high risk or low risk allele. Secondly, homozygote comparison was used to find out the high risk or low risk genotype. The last pattern is comparing homozygote genotype versus allele carriers. In the present study, the moderate-significant heterogeneity between studies occurred in the overall population. Common reasons for heterogeneity consist of differences in the investigated populations or in genotyping methods or in sample size or it may be derived from other risk factors. By performing meta-regression, and subgroup analysis, we found that ethnicity might contribute substantial heterogeneity to final results. By Galbraith plot analysis, we found the studies Liu et al. and Hamdy et al. were not within reasonable limits. Then we explored the two studies carefully and discovered their shortcomings. The $P$ value $<0.05$ of HWE in control group was found in literature of Liu et al. And we found the sample size $<100$ participants in literature of Hamdy et al. The results of our meta-analysis were not altered by omitting the two studies. The results of sensitive analysis and publication bias demonstrated that the results of our meta-analysis were stable and reliable.

Although the present meta-analysis is comprehensive and rigorous, there are still some disadvantages existing. Firstly, more studies with different ethnicities are also needed because different ethnicities have different genetic backgrounds. Various ethnicities should be investigated and discussed including African population, Asian population, mixed population and Caucasian population. Secondly, different kinds of confounding factors such as age, gender and radiation exposure are not taken into consideration due to limited dataset $[44,45]$. Therefore, more studies in the future on XRCC3 gene considering all of these factors should be performed for subgroup analysis [46, 47]. Thirdly, the relevant GWAS has not been investigated. Thus, rigorous GWAS should be performed for further trans-ethnic and trans-trait metaanalysis [48]. Lastly, if many independent SNPs in other genome regions, XRCC3 Thr241Met polymorphism and environmental factors can be precisely explored, maybe we can establish a machine-learning prediction model, which contributes to early diagnosis for multiple diseases $[49,50]$.

\section{Conclusions}

The present meta-analysis suggests that the XRCC3 is a candidate gene for leukemia susceptibility. The XRCC3 Thr241Met polymorphism may be risk factor for leukemia in Caucasian population. Further studies investigating other confirmed genetic factors and possible gene-gene and gene-environmental interactions for XRCC3 Thr241Met polymorphism should be performed.

\section{Abbreviations}

PB: Population-based; HWE: Hardy-Weinberg equilibrium; RFLP: Restricted fragment length polymorphism; NOS: Newcastle-Ottawa Score; OR: Odds ratio; 95\% Cl: 95\% Confidence interval.

\section{Supplementary Information}

The online version contains supplementary material available at https://doi. org/10.1186/s12920-021-01076-w.

\section{Additional file 1: Table S1. PRISMA 2009 checklist \\ Additional file 2: Table S2. PRISMA 2009 Flow Diagram.}

Additional file 3: Figure S3. Results of evaluating publication bias of other four genetic models (A: homozygote comparison, B: heterozygous comparison, C: recessive genetic model, D: dominate genetic model).

\section{Acknowledgements \\ None.}

\section{Authors' contributions}

Conceptualization: ZX, WP. Data curation: WP, QL, WC. Formal analysis: WP, QL, WC. Funding acquisition: ZX, QL, WC, XZ. Investigation: ZX, QL, WC, XZ. Methodology: ZX, QL, WC, XZ. Project administration: ZX, WP. Resources: QL, WC, XZ. Software: QL, WC, XZ. Supervision: ZX, QL, WC, XZ. Validation: ZX, WP, QL, WC, XZ. Visualization: ZX. Writing—original draft: ZX, WP, QL, WC, XZ. Writing-review and editing: ZX,WP, QL, WC, XZ. All authors read and approved the final manuscript.

\section{Funding}

This work was supported by Scientific Research Foundation for Doctors of Zunyi Medical College (BS2018-01).

\section{Availability of data and materials}

The datasets used and/or analyzed during the current study are available from the corresponding author on reasonable request.

\section{Declarations}

Ethics approval and consent to participate

Not applicable.

Consent for publication

Not applicable. 


\section{Competing interests}

The authors declare that they have no competing interests.

Received: 15 April 2021 Accepted: 30 August 2021 Published online: 18 September 2021

\section{References}

1. Bray F, Ferlay J, Soerjomataram I, Siegel RL, Torre LA, Jemal A. Global cancer statistics 2018: GLOBOCAN estimates of incidence and mortality worldwide for 36 cancers in 185 countries. CA Cancer J Clin. 2018;68(6):394-424.

2. Liu B, Liu QM, Li HZ, Ren YJ, Zhang Y, Du LB. Analysis of the incidence and mortality of leukemia in the cancer registration area of Zhejiang Province from 2010 to 2014. Zhonghua Yu Fang Yi Xue Za Zhi. 2019;53(11):1158-61.

3. Gross SA, Paustenbach DJ. Shanghai Health Study (2001-2009): what was learned about benzene health effects? Crit Rev Toxicol. 2018;48(3):217-51.

4. Teras LR, Diver WR, Deubler EL, Krewski D, Flowers CR, Switchenko $J M$, et al. Residential ambient benzene exposure in the United States and subsequent risk of hematologic malignancies. Int J Cancer. 2019;145(10):2647-60.

5. Rauscher GH, Shore D, Sandler DP. Hair dye use and risk of adult acute leukemia. Am J Epidemiol. 2004;160(1):19-25.

6. Bolufer P, Collado M, Barragan E, Cervera J, Calasanz MJ, Colomer D, et al. The potential effect of gender in combination with common genetic polymorphisms of drug-metabolizing enzymes on the risk of developing acute leukemia. Haematologica. 2007;92(3):308-14.

7. de Boer JG. Polymorphisms in DNA repair and environmental interactions. Mutat Res. 2002;509(1-2):201-10.

8. Jackson SP. Sensing and repairing DNA double-strand breaks. Carcinogenesis. 2002;23(5):687-96.

9. Kawabata M, Kawabata T, Nishibori M. Role of recA/RAD51 family proteins in mammals. Acta Med Okayama. 2005;59(1):1-9.

10. Kurumizaka H, Enomoto R, Nakada M, Eda K, Yokoyama S, Shibata T. Region and amino acid residues required for Rad51C binding in the human Xrcc3 protein. Nucleic Acids Res. 2003;31(14):4041-50.

11. Yan Y, Liang H, Li T, Guo S, Li M, Qin X, et al. Association of XRCC3 Thr241Met polymorphism and leukemia risk: evidence from a metaanalysis. Leuk Lymphoma. 2014;55(9):2130-4.

12. Qin L, Chen X, Li P, Yang Z, Mo W. Comprehensive assessment of the association between DNA repair gene XRCC3 Thr241Met polymorphism and leukemia risk. Tumour Biol. 2014;35(3):2521-8.

13. Higgins JP, Thompson SG, Deeks JJ, Altman DG. Measuring inconsistency in meta-analyses. BMJ. 2003;327(7414):557-60.

14. DerSimonian R, Laird N. Meta-analysis in clinical trials. Control Clin Trials. 1986;7(3):177-88.

15. Mantel N, Haenszel W. Statistical aspects of the analysis of data from retrospective studies of disease. J Natl Cancer Inst. 1959;22(4):719-48.

16. Begg CB, Mazumdar M. Operating characteristics of a rank correlation test for publication bias. Biometrics. 1994;50(4):1088-101.

17. Page MJ, Moher D, Bossuyt PM, Boutron I, Hoffmann TC, Mulrow CD, et al. PRISMA 2020 explanation and elaboration: updated guidance and exemplars for reporting systematic reviews. BMJ. 2021;372:n160.

18. Page MJ, McKenzie JE, Bossuyt PM, Boutron I, Hoffmann TC, Mulrow CD, et al. The PRISMA 2020 statement: an updated guideline for reporting systematic reviews. BMJ. 2021;372:n71.

19. Seedhouse C, Bainton R, Lewis M, Harding A, Russell N, Das-Gupta E. The genotype distribution of the XRCC1 gene indicates a role for base excision repair in the development of therapy-related acute myeloblastic leukemia. Blood. 2002;100(10):3761-6.

20. Seedhouse C, Faulkner R, Ashraf N, Das-Gupta E, Russell N. Polymorphisms in genes involved in homologous recombination repair interact to increase the risk of developing acute myeloid leukemia. Clin Cancer Res. 2004;10(8):2675-80.

21. Matullo G, Dunning AM, Guarrera S, Baynes C, Polidoro S, Garte S, et al. DNA repair polymorphisms and cancer risk in non-smokers in a cohort study. Carcinogenesis. 2006;27(5):997-1007.
22. Bhatla D, Gerbing RB, Alonzo TA, Mehta PA, Deal K, Elliott J, et al. DNA repair polymorphisms and outcome of chemotherapy for acute myelogenous leukemia: a report from the Children's Oncology Group. Leukemia. 2008;22(2):265-72.

23. Zhang ZQ, Yang L, Zhang Y, Yang YH, Nie L, Li L, et al. Relationship between NQO1C(609T), RAD51(G135C), XRCC3(C241T) single nucleotide polymorphisms and acute lymphoblastic leukemia. Zhongguo Shi Yan Xue Ye Xue Za Zhi. 2009;17(3):523-8.

24. Hamdy MS, El-Haddad AM, Bahaa El-Din NM, Makhlouf MM, Abdel-Hamid SM. RAD51 and XRCC3 gene polymorphisms and the risk of developing acute myeloid leukemia. J Investig Med. 2011;59(7):1124-30.

25. Liu L, Yang L, Mi Y, Wang J, Li J, Zhang Y, et al. RAD51 and XRCC3 polymorphisms: impact on the risk and treatment outcomes of de novo inv(16) or t(16;16)/CBFbeta-MYH11(+) acute myeloid leukemia. Leuk Res. 2011;35(8):1020-6.

26. Abramenko I, Bilous N, Chumak A, Kostin A, Martina Z, Dyagil I. DNA repair polymorphisms in B-cell chronic lymphocytic leukemia in sufferers of Chernobyl Nuclear Power Plant accident. J Radiat Res. 2012;53(3):497-503.

27. Erculj N, Faganel Kotnik B, Debeljak M, Jazbec J, Dolzan V. DNA repair polymorphisms influence the risk of second neoplasm after treatment of childhood acute lymphoblastic leukemia. J Cancer Res Clin Oncol. 2012;138(11):1919-30.

28. Banescu C, Tilinca M, Benedek EL, Demian S, Macarie I, Duicu C, et al. XRCC3 Thr241Met polymorphism and risk of acute myeloid leukemia in a Romanian population. Gene. 2013;526(2):478-83.

29. Sorour A, Ayad MW, Kassem H. The genotype distribution of the XRCC1, XRCC3, and XPD DNA repair genes and their role for the development of acute myeloblastic leukemia. Genet Test Mol Biomark. 2013:17(3):195-201.

30. Banescu C, Trifa AP, Demian S, Benedek Lazar E, Dima D, Duicu C, et al. Polymorphism of XRCC1, XRCC3, and XPD genes and risk of chronic myeloid leukemia. Biomed Res Int. 2014;2014:213790.

31. Smolkova B, Dusinska M, Hemminki K. NBN and XRCC3 genetic variants in childhood acute lymphoblastic leukaemia. Cancer Epidemiol. 2014;38(5):563-8.

32. Miao L, Qian XF, Yang GH, Zhao LD. Relationship between RAD51G135C and XRCC3-C241T single nucleotide polymorphisms and onset of acute myeloid leukemia. Zhongguo Shi Yan Xue Ye Xue Za Zhi. 2015;23(3):605-11.

33. Mutlu P, Elci MP, Yildirim M, Nevruz O, Cetin AT, Avcu F. Identification of XRCC1 Arg399GIn and XRCC3 Thr241Met polymorphisms in a Turkish population and their association with the risk of chronic lymphocytic leukemia. Indian J Hematol Blood Transfus. 2015;31(3):332-8.

34. Pei JS, Chang WS, Hsu PC, Chen CC, Cheng SP, Wang YC, et al. The contribution of XRCC3 genotypes to childhood acute lymphoblastic leukemia. Cancer Manag Res. 2018;10:5677-84.

35. Chen BA, Huang ZH, Zhang XP, Ou-Yang J, Li JY, Zhai YP, et al. An epidemiological investigation of leukemia incidence between 2003 and 2007 in Nanjing. China J Hematol Oncol. 2010;3:21.

36. Swaminathan R, Rama R, Shanta V. Childhood cancers in Chennai, India, 1990-2001: incidence and survival. Int J Cancer. 2008;122(11):2607-11.

37. Kamsa-ard S, Wiangnon S, Kamsa-ard S, Suwanrungruang K, Jetsrisuparb A, Horsith S. Trends in incidence of childhood leukemia, Khon Kaen, Thailand, 1985-2002. Asian Pac J Cancer Prev. 2006;7(1):75-8.

38. Karimi M, Yarmohammadi H, Sabri MR. An analysis of prognostic factors and the five-year survival rate in childhood acute lymphoblastic leukemia. Med Sci Monit. 2002;8(12):CR792-6.

39. Bhurgri Y. Childhood lymphoma and leukaemia. J Pak Med Assoc. 2006;56(4):147-8.

40. Pui CH, Relling MV, Sandlund JT, Downing JR, Campana D, Evans WE. Rationale and design of Total Therapy Study XV for newly diagnosed childhood acute lymphoblastic leukemia. Ann Hematol. 2004;83(Suppl 1):S124-6.

41. Chinese Journal of $C$. The 150 most important questions in cancer research and clinical oncology series: questions 86-93: Edited by Chinese Journal of Cancer. Chin J Cancer. 2018;37(1):1.

42. Katsimpardi K, Papadakis V, Pangalis A, Parcharidou A, Panagiotou $J P$, Soutis $M$, et al. Infections in a pediatric patient cohort with acute 
lymphoblastic leukemia during the entire course of treatment. Support Care Cancer. 2006;14(3):277-84.

43. Jin $G, X u M$, Zou M, Duan S. The processing, gene regulation, biological functions, and clinical relevance of N4-acetylcytidine on RNA: a systematic review. Mol Ther Nucleic Acids. 2020;20:13-24.

44. Zhou X, Li Q, Xu J, Zhang X, Zhang H, Xiang Y, et al. The aberrantly expressed miR-193b-3p contributes to preeclampsia through regulating transforming growth factor-beta signaling. Sci Rep. 2016;6:19910.

45. Yan X, Zhao X, Li J, He L, Xu M. Effects of early-life malnutrition on neurodevelopment and neuropsychiatric disorders and the potential mechanisms. Prog Neuropsychopharmacol Biol Psychiatry. 2018;83:64-75.

46. Jiang L, Wang K, Lo K, Zhong Y, Yang A, Fang $X$, et al. Sex-specific association of circulating ferritin level and risk of type 2 diabetes: a doseresponse meta-analysis of prospective studies. J Clin Endocrinol Metab. 2019;104(10):4539-51

47. $X u M Q, Y e Z, H u F B, H e L$. Quantitative assessment of the effect of angiotensinogen gene polymorphisms on the risk of coronary heart disease. Circulation. 2007;116(12):1356-66.
48. Wu Y, Cao H, Baranova A, Huang H, Li S, Cai L, et al. Multi-trait analysis for genome-wide association study of five psychiatric disorders. Trans Psychiatry. 2020;10(1):209.

49. Liu M, Li F, Yan H, Wang K, Ma Y, Alzheimer's Disease Neuroimaging I, et al. A multi-model deep convolutional neural network for automatic hippocampus segmentation and classification in Alzheimer's disease. Neuroimage. 2020;208:116459.

50. Yu H, Pan R, Qi Y, Zheng Z, Li J, Li H, et al. LEPR hypomethylation is significantly associated with gastric cancer in males. Exp Mol Pathol. 2020;116:104493.

\section{Publisher's Note}

Springer Nature remains neutral with regard to jurisdictional claims in published maps and institutional affiliations.
Ready to submit your research? Choose BMC and benefit from:

- fast, convenient online submission

- thorough peer review by experienced researchers in your field

- rapid publication on acceptance

- support for research data, including large and complex data types

- gold Open Access which fosters wider collaboration and increased citations

- maximum visibility for your research: over $100 \mathrm{M}$ website views per year

At BMC, research is always in progress.

Learn more biomedcentral.com/submissions 